\title{
Parameter Sensitivity Analysis for Design and Control of Force Transmission Systems
}

\author{
Vincent Hayward and Juan Manuel Cruz-Hernández \\ McGill University \\ Center for Intelligent Machines \\ 3480 University Street \\ Montréal, Québec, Canada, H3A 2A7
}

\begin{abstract}
We apply sensitivity analysis to the design and control of force transmission systems. With this approach, the effect of the values of the system parameters on the response can be investigated: transmission ratio, sensor placement, damping and allowable load variation. It is found that feedback must be applied to reduce the sensitivity of the system response to the load. Based on these observations, a loop shaped feedback compensator design is proposed. Such compensators can compensate for the nonlinear behavior of the transmissions due to friction, while exhibiting good disturbance rejection and robustness. This is achieved without detailed knowledge of friction behavior and without measurement nor estimation of velocity. Experimental results using a test bench are discussed.
\end{abstract}

\section{Introduction}

There is some freedom in the design of force transmission systems. The question arises of how to choose certain design parameters to improve performance: transmission ratio, sensor placement, damping, and allowable load variation. Sensitivity analysis is applied to express the influence of these parameters on the system response in open and closed loop. The objectives include precise control of force, extension of the frequency response to the widest range possible, and reduction of friction and inertia as experienced from the load side of the transmission.

It is found in particular that the response is highly sensitive to the load variations, so a compensator design method is proposed to reduce this sensitivity and which considers friction as a disturbance to be rejected. This scheme, based on loop shaping, can be applied to a wide class of systems and neither rely on a detailed knowledge of the nonlinear behavior of friction, nor requires measurement of velocity.

Background material on sensitivity analysis can be found in (Frank, 1978) and (Wierzbicki, 1984). Sensitivity analysis provides the basic methods for studying the sensitivity of a system to 
ASME J. of Dynamics Systems, Meas. and Contr. 120(2):241--249. 1998.

parameter variations. Here, the system is described by a linear transfer function $G=G(s, \alpha)$, where the plant response varies not only with $s$, but also with parameters $\alpha=\left[\alpha_{1}, \ldots, \alpha_{n}\right]^{T}$. These parameters can be for example initial conditions, natural frequencies, time invariant or time variant coefficients, etc. We will use the Bode Sensitivity Function defined as the logarithmic partial derivative of $G$ with respect to the parameter $\alpha_{i}$, defined as:

$$
\left.\mathbf{S}_{\alpha_{i}}^{G}(s) \triangleq \frac{\partial \ln G(s, \alpha)}{\partial \ln \alpha_{i}}\right|_{\alpha_{0}}=\left.\frac{\partial G / G}{\partial \alpha_{i} / \alpha_{i}}\right|_{\alpha_{0}}=\left.\frac{\partial G(s, \alpha)}{\partial \alpha_{i}}\right|_{\alpha_{0}} \frac{\alpha_{0}}{G\left(s, \alpha_{0}\right)},
$$

and $G(s)\left(1 \pm S_{\alpha_{i}}^{G}(s)\right)$ will represent the range of possible transfer functions under the variation of parameter $\alpha_{i}$.

Sensitivity analysis has been used in several areas of engineering. It has been applied, for example, to the stability analysis of robot control (e.g. Kazerooni, 1988), modal analysis in a three-link articulated robot manipulator (Jen and Johnson, 1991), and in the optimal design of robot mass parameters (Guo and Zhang, 1988).

\section{Model}

Figure 1 illustrates an engineering model of most force transmissions. It includes the inertia of the motor $I_{M}$, linear damping lumped into damper $B, r$ the transmission ratio, $k_{1}$ the elasticity of the proximal section of the transmission, $k_{2}$ the elasticity of the distal part, $I_{c}$ the inertia of the driven portion of the transmission, and $Z_{H}$ an arbitrary impedance representing the load. The total elasticity

$$
k_{e}=\left(k_{1} k_{2}\right) /\left(k_{1}+k_{2}\right),
$$

expresses the degree of "collocation" of the force measurement. When the force measurement is taken at the output of the primary actuator, $k_{2} \ll k_{1}$ so $k_{e} \simeq k_{2}$. Inversely, $k_{e} \simeq k_{1}$ when the measurement is taken near the load.

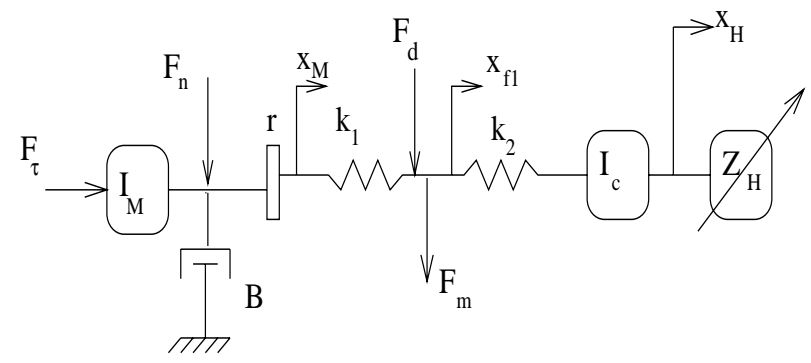

Figure 1: Model of the plant.

The relevant signals are $F_{\tau}$ the force produced by the actuator, $F_{n}$ a disturbance force signal representing friction in the motor, $F_{d}$ another disturbance signal representing the friction in the transmission, and one sensed signal $F_{m}$, the force measured at some intermediate location between the actuator and the load.

The objective is to transmit a force applied to the load, consequently precise control would require the force sensor to be placed near it. Unfortunately, sensor placement has several effects 
ASME J. of Dynamics Systems, Meas. and Contr. 120(2):241--249. 1998.

on the closed loop system response, so it must be considered carefully as will be discussed in Section 3.

Looking at the transmission of force from the actuator to the sensor, transfer functions can be worked out for both the actuator force and the actuator friction signal.

$$
\begin{aligned}
G(s) & =\frac{F_{m}}{F_{\tau}}=-\frac{F_{m}}{F_{n}}=\frac{N(s)}{D(s)} \\
& =\frac{k_{e} I_{c} s^{2}+k_{z} k_{e}}{r^{2} I_{M} I_{c} s^{4}+r^{2} I_{c} B s^{3}+\left(k_{e} I c+r^{2} I_{M}\left(k_{z}+k_{e}\right)\right) s^{2}+r^{2} B\left(k_{e}+k_{z}\right) s+k_{z} k_{e}}
\end{aligned}
$$

In the above expression and in the rest of the paper, the variable load is simplified to a variable elasticity $k_{z}$, which is a worst case as far as control stability is concerned. The negative sign of the transfer function $F_{m} / F_{n}$ accounts for the fact that $F_{\tau}$ and $F_{n}$ are applied in opposite directions.

The transfer function from the disturbance friction signal due to the transmission to the sensor is:

$$
\frac{F_{m}}{F_{d}}=\frac{r^{2} I_{M} I_{c}}{D(s)}
$$

\section{Analysis}

\section{$3.1 \quad$ Effect of $r$}

The effect of $r$ can be found by inspection of the transfer functions (3) and (4). The factor $r^{2}$ in (3) affects both the inertia and the damping of the transmission as experienced from the output because both result from a force divided by a time derivative of a displacement. This quadratic dependency is an important consideration since the open loop bandwidth will drop dramatically with an increase of $r$. Friction will increase in open loop linearly only with $r$ under the assumption that it is a rate independent signal.

The numerator of the disturbance transfer function (4) depends quadratically on $r$ but does not depend on frequency. The higher the actuator inertia is, and the higher the transmission ratio is, the better the friction disturbance rejection is. In other terms, for a given desired output signal and a given disturbance, an actuator with higher inertia (or a higher transmission ratio) will demand a larger input signal, improving the signal-to-disturbance ratio.

In closed loop the apparent inertia is divided by the loop gain. The ratio $r$ has an effect on the highest achievable loop gain given a desired phase margin, since $r$ also appears in the denominator of the transfer function.

\subsection{Effect of $k_{e}$}

To understand the effect of the sensor placement, equation (1) is applied to compute the sensitivity function of $G$ respect to $k_{e}$,

$$
S_{k_{e}}^{G}(s)=\frac{r^{2} I_{M} I_{c} s^{4}+r^{2} I_{c} B s^{3}+r^{2} I_{M} k_{z} s^{2}+r^{2} B k_{z} s}{r^{2} I_{M} I_{c} s^{4}+r^{2} I_{c} B s^{3}+\left(k_{e} I c+r^{2} I_{M}\left(k_{z}+k_{e}\right)\right) s^{2}+r^{2} B\left(k_{e}+k_{z}\right) s+k_{z} k_{e}},
$$


ASME J. of Dynamics Systems, Meas. and Contr. 120(2):241--249. 1998.

which in turns depends on $k_{1}$ and $k_{2}$ :

$$
S_{k_{1}}^{G}(s)=S_{k_{e}}^{G}(s) \frac{k_{2}}{k_{1}+k_{2}}, \quad S_{k_{2}}^{G}(s)=S_{k_{e}}^{G}(s) \frac{k_{1}}{k_{1}+k_{2}} .
$$

While there is no value in analysing the plant when the values of $k_{1}$ or $k_{2}$ approach zero or infinity, it is possible to analyze sensitivity when one value is kept fixed and the other varied as in Figure 2 where $k_{2}$ is large. Using parameter values close to those of an actual prototype $\left(B=4, k_{z}=1 \times 10^{9}, r=10, I m=12.8 \times 10^{-6}, I c=0.0235176, k 2=2.566 \times 10^{4}\right)$ and different values for $k_{1}\left(k_{1} \in\left\{1 \times 10^{3}, 2.566 \times 10^{4}, 4.56 \times 10^{6}\right\}\right)$, Figure 2 was obtained.

Two effects are observed: (a) the bandwidth of the system increases with $k_{1}$ for a fixed $k_{2}$ in the high frequency region; (b) the sensitivity to variation of $k_{1}$ changes widely. (For a small $k_{2}$, the bandwidth variations would be limited to the low frequency region and the sensitivity would always be low.) All cases are summarized in the table 1. When one of the parameters is small the sensitivity of the plant respect to that parameter is small, if its value is large then the the system is not sensitive to that parameter.

\begin{tabular}{|c|c|c|}
\hline$k_{1}$ & $k_{2}$ & \multicolumn{1}{c|}{ Sensitivity } \\
\hline \hline small & small & $\begin{array}{l}\cdot \text { very sensitive to small } \\
\text { changes in } k_{1} \text { or } k_{2} \\
\text { i.e. } S_{k_{1}}^{G} \rightarrow 1 \text { and } S_{k_{2}}^{G} \rightarrow 1\end{array}$ \\
\hline small & large & $\begin{array}{l}\text { - very sensitive to small } \\
\text { changes in } k_{1} \text { i.e. } S_{k_{1}}^{G} \rightarrow 1 \\
\cdot \text { not sensitive to } \\
\text { changes in } k_{2} \text { i.e. } S_{k_{2}}^{G} \rightarrow 0\end{array}$ \\
\hline large & small & $\begin{array}{l}\cdot \text { very sensitive to small } \\
\text { changes in } k_{2} \text { i.e. } S_{k_{2}}^{G} \rightarrow 1 \\
\cdot \text { not sensitive to } \\
\text { changes in } k_{1} \text { i.e. } S_{k_{1}}^{G} \rightarrow 0\end{array}$ \\
\hline large & large & $\begin{array}{l}\cdot \text { not sensitive to } \\
\text { changes in } k_{1} \text { or } k_{2} \\
\text { i.e. } S_{k_{1}}^{G} \rightarrow 0 \text { and } S_{k_{2}}^{G} \rightarrow 0\end{array}$ \\
\hline
\end{tabular}

Table 1: Comparison of the sensitivity function for different values of $k_{1}$ and $k_{2}$

In practice, stiffness is always limited by functional considerations, so the designer must choose where to place the sensor (choosing between line 2 and 3 of Table 1). Figure 2.a shows that a soft transmission with a stiff end portion $\left(k_{1} \ll k_{2}\right)$ is very sensitive in the high frequencies to slight changes in $k_{1}$, while a stiff transmission with a soft end-portion, not only increases the bandwidth of the response but also decreases the sensitivity to very small values, as on Figure 2.c. Therefore, as mentioned before, precise control is in conflict with bandwidth and robustness. It can be concluded that $k_{e}$ expressing the degree of collocation of the force sensor has a major influence on the ability for a transmission to be force-controlled.

This $k_{e}$-dependence has been noticed by many researchers while implementing force control on a manipulator (Eppinger and Seering, 1987): a stiff force sensor clamped at the wrist and 
ASME J. of Dynamics Systems, Meas. and Contr. 120(2):241--249. 1998.

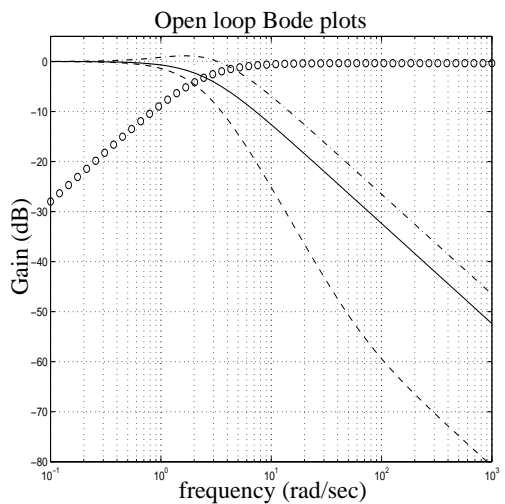

a)

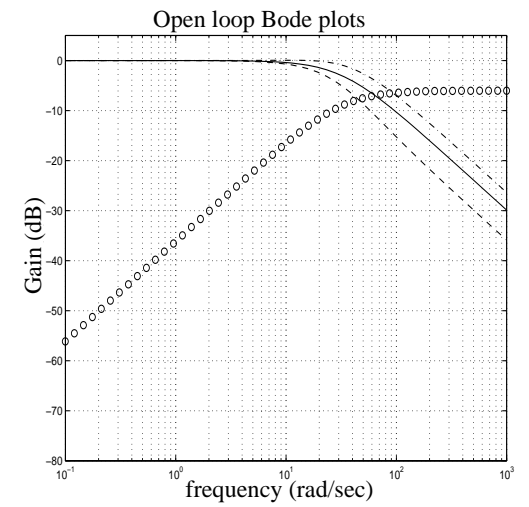

b)

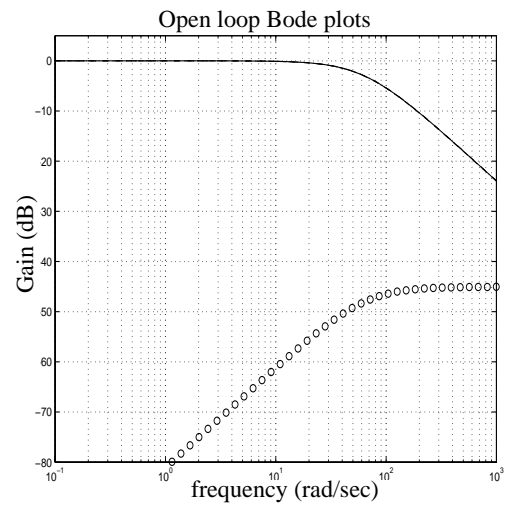

c)

Figure 2: Plots for the transfer function, sensitivity function, and $G(s)\left(1 \pm S_{k_{1}}^{G}(s)\right)$ with respect to $k_{1}$. Notation: Sensitivity function $S_{k_{1}}^{G}(s) \circ \circ \circ$, Transfer Function $G(s)-, G(s)\left(1+S_{k_{1}}^{G}(s)\right)-\cdot-\cdot-\cdot, G(s)\left(1-S_{k_{1}}^{G}(s)\right)---$, a) $k_{1}=1.0 \times 10^{3}$, b) $k_{1}=2.6 \times 10^{4}$, c) $k_{1}=4.6 \times 10^{6}$

separated from the actuator by a soft transmission will make the control difficult, and if at all possible, the response will either be highly sensitive to the load variations (hence the hard contact bouncing so often discussed) or effective only in the very lowest range of the frequency domain. A force sensor located near the actuator and separated from the load by the structural elasticity of the manipulator has exactly the opposite property: the sensitivity to the load is low (so a single tuning will work for a wide range of loads but disturbance rejection is less good so it cannot be precise) and the response range is wide.

A parallel can be drawn between the effect of a gear ratio for position control and the effect of $k_{e}$ in force control. A high gear ratio makes the position control insensitive to load variations and other disturbances (so it is easy to control), while a direct drive robot will be maximally sensitive to the same factors (so it can be accurate and the disturbance rejection can be good but is hard to control). Therefore, the collocation factor $k_{e}$ plays for force control a role analogous to $r$ for position control.

\subsection{Effect of $B$}

This effect of damping $B$ on the properties of the transmission are now studied. Consider $S_{B}^{G}$ given by

$$
S_{B}^{G}(s)=\frac{-B r^{2} s\left(I_{c} s^{2}+\left(k_{e}+k_{z}\right)\right)}{r^{2} I_{M} I_{c} s^{4}+r^{2} I_{c} B s^{3}+\left(k_{e} I c+r^{2} I_{M}\left(k_{z}+k_{e}\right)\right) s^{2}+r^{2} B\left(k_{e}+k_{z}\right) s+k_{z} k_{e}}
$$

The sensitivity curves for the nominal plant parameter values are similar to the curves produced by $S_{k_{1}}^{G}$, so they are not reproduced here. The variations of $B$ affect the bandwidth of G(s) in the same way the variations of $k_{1}$ affect it, in fact: $S_{B}^{G}=S_{k_{1}}^{G}\left(-B r^{2} s\right)$.

However, $\lim _{B \rightarrow 0} S_{k_{1}}^{G}=0$ and $\lim _{B \rightarrow \infty} S_{k_{1}}^{G}=0$. For a soft transmission with a stiff end portion, the plant response is mostly affected in the vicinity of the cut-off frequency (where half of the input signal is dissipated). Damping should be low. If it is high, small changes will 
ASME J. of Dynamics Systems, Meas. and Contr. 120(2):241--249. 1998.

have large effects on the plant's response, possibly unpredictably destabilizing the closed loop response.

\subsection{Effect of $k_{z}$}

We now consider the effect of load changes on the response.

$$
S_{k_{z}}^{G}(s)=\frac{k_{z}\left(k_{e}^{2} r^{2} I_{M} s^{2}+k_{e}^{2} r^{2} B s\right)}{\frac{r^{2} I_{M} I_{c}^{2} s^{6}+r^{2} I_{c}^{2} B s^{5}+\left(k_{e} I_{c}\left(k_{e} I_{c}+r^{2} I_{M}\left(k_{e}+2 k_{z}\right)\right)\right) s^{4}+r^{2} B k_{e} I_{c}\left(k_{e}+\right.}{\left.+2 k_{z}\right) s^{3}+k_{z} k_{e}\left(2 I_{c} k_{e}+r^{2} I_{M}\left(k_{z}+k_{e}\right)\right) s^{2}+r^{2} B k_{z} k_{e}\left(k_{e}+k_{z}\right) s+k_{z}^{2} k_{e}^{2}}}
$$

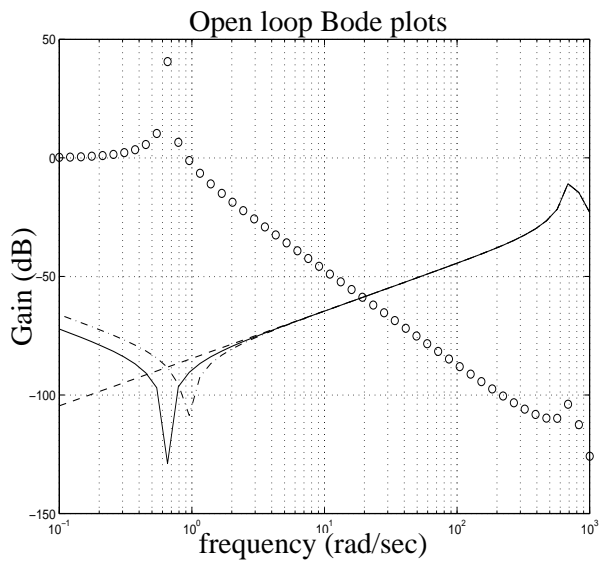

a)

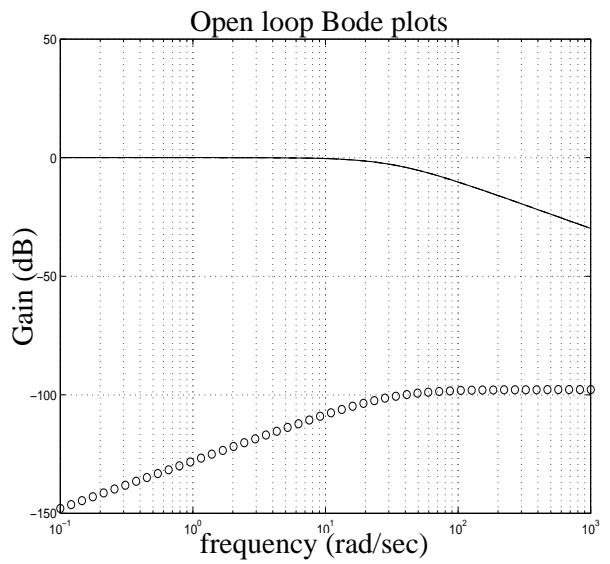

b)

Figure 3: Transfer function, sensitivity function, and $G(s)\left(1 \pm S_{k_{z}}^{G}(s)\right)$ with respect to $k_{z}$.

Notation: Sensitivity function $S(s) \circ \circ \circ$, Transfer Function $G(s)-, G(s)\left(1+S_{k_{z}}^{G}(s)\right)-\cdot-\cdot-\cdot, G(s)\left(1-S_{k_{z}}^{G}(s)\right)$ --- a) $k_{z}=0.01$, b) $k_{z}=1.0 \times 10^{9}$

Stiffness values cannot be chosen to be zero or infinity since they would lead to an absence of transmission. Sensitivity is studied for very large and very small values.

Figure 3 shows that the sensitivity to this parameter is very high for small values, while it vanishes at high values. The sensitivity has a resonant shape with the peak in the vicinity of the plant's first natural resonance. In (3), the independent term, both in the numerator and the denominator, depends on $k_{z}$, so if $k_{z}$ is small, the plant will have two zeros and one pole. This means that the response is essentially unknown when the load is soft so the transmission is unable to apply precise forces to the load in that case.

\subsection{Effect of Feedback}

Feedback has an effect on the sensitivity to $B$ and to $k_{z}$. These parameters are not freely chosen in the design of transmission: $k_{z}$ is unknown, and $B$ should be set as low as technology allows. In contrast, $r$ and $k_{e}$ are free design parameters which affect what can be done by feedback. In order to investigate the effect of feedback on sensitivity, a proportional controller of gain $K$ 
ASME J. of Dynamics Systems, Meas. and Contr. 120(2):241--249. 1998.

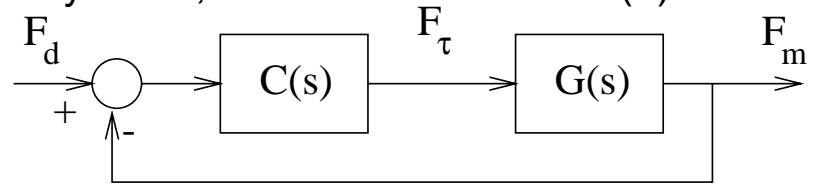

Figure 4: Feedback connection of the plan $G(s)$ and the controller $C(s)$.

is analyzed because of its simplicity (See Figure 4, where $C(s)=K$ ). The closed loop transfer function $T(s)=\frac{K G(s)}{1+K G(s)}$, from $F_{d}$ (desired force) to $F_{m}$ (measured force), is:

$$
T(s)=\frac{\left(k_{e} I_{c} s^{2}+k_{z} k_{e}\right) K}{r^{2} I_{M} I_{c} s^{4}+r^{2} I_{c} B s^{3}+\left(k_{e} I c(1+K)+r^{2} I_{M}\left(k_{z}+k_{e}\right)\right) s^{2}+r^{2} B\left(k_{e}+k_{z}\right) s+k_{z} k_{e}(1+K)}
$$

For a closed loop transfer function, where $\alpha$ is some parameter under study, $S_{\alpha}^{T}=S_{G}^{T} S_{\alpha}^{G}$. Since $S_{\alpha}^{G}$ was computed for $B$ and $k_{z}$ we only need to compute $S_{G}^{T}$. Setting $C(s)=K$ leads to $S_{G}^{T}=1 /(1+K G)$. To reduce the sensitivity with respect to parameter $B$ or $k_{z}$ we need to minimize $S_{G}^{T}$ by setting $K$ to some optimum value. Since the denominator of $S_{G}^{T}$ is the same as $T(s)$, a value for $K$ must be found that will also not only preserve stability but achieve a desired stability margin.

The choice of $K$ only affects sensitivity with respect to $B$ beyond the cut-off frequency of the closed loop system. For $k_{z}$ sensibility, Figure 5 shows that for a small value, the single gain feedback controller neither improves the response, nor sensitivity. It is therefore concluded that more elaborate controllers must be considered.

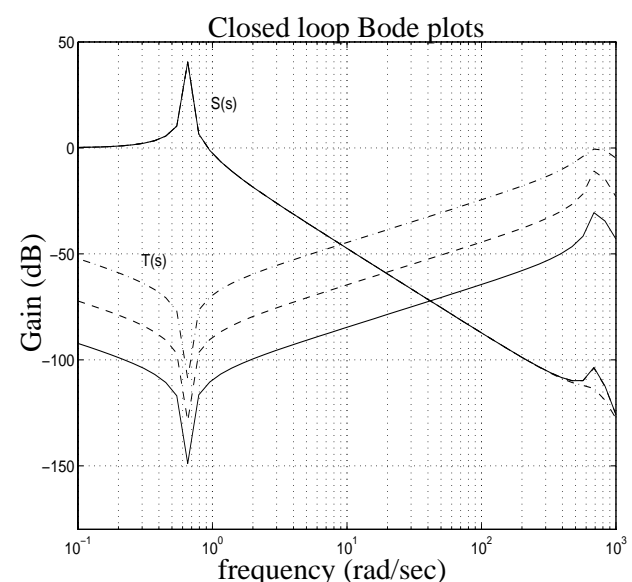

a)

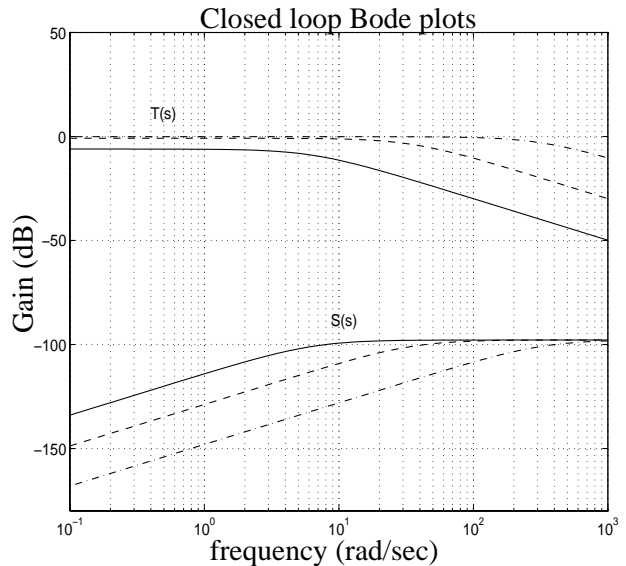

b)

Figure 5: Plots for the transfer function $\mathrm{T}(\mathrm{s})$ and sensitivity function $S_{k_{z}}^{G}(s)$ respect to $k_{z}$. a) $k_{z}=0.01$, and b) $k_{z}=10^{9}$ for a constant controller with values $1,10,100$. Notation: $K=1-, K=10---$, $K=100-\cdot-\cdot-$.

We consider the next level in complexity which could be a lead compensator of the form $C(s)=(s+z) /(s+p)$. It is found that the sensitivity function becomes:

$$
S_{G}^{T}(s)=\frac{(s+p) D(s)}{(s+p) D(s)+(s+z) N(s)}
$$


ASME J. of Dynamics Systems, Meas. and Contr. 120(2):241--249. 1998.

Any change in $p$ or $z$ will affect sensitivity in the same order as a simple gain $K$ with the disadvantage of complicating the tuning.

In the absence of further information about the exact nature of the plant, the simple gain controller $K$ should be preferred over a complex controller. This simple controller will improve the response, and decrease the sensitivity to high $k_{z}$ 's, while its tuning is particularly simple. It will only involve raising the value of $K$ under the worse stability conditions (smallest needed $k_{z}$ ) until the closed loop stability is compromised, while observing the response in the time domain, for example.

The loopshaping control design technique will be shown in the next section to result in a controller capable of a large reduction in the $k_{z}$ sensitivity, even for small values because it will result in a stiffening of the plant, making it less sensitive to load variations, as well as in the cancellation of its resonant characteristic.

\section{Controller Design on a Case Study}

We now design a controller for a tendon transmission. A tendon transmission has by nature a significant amount of elasticity and therefore is subject to the previous analysis. The objectives are to improve the extent and the precision of the response, to reduce the apparent friction of the transmission when it is back driven, to reduce its apparent inertia, and to reduce sensitivity to load variations.

\subsection{Tendon Transmission}

The purpose of the tendon drive is to transmit mechanical signals from a remote location, so that the actuators can be mechanically grounded and large amounts of power can be transmitted by small amounts of material.

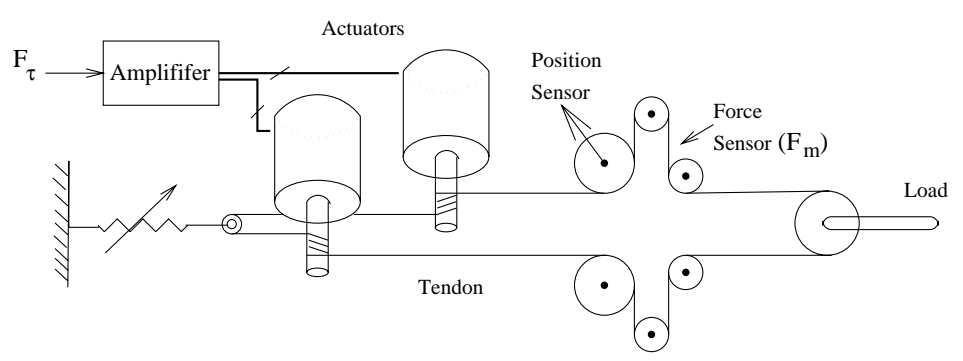

Figure 6: Transmission structure.

The transmission is of type $2 \mathrm{~N}$ (Jacobsen et al., 1989), with two actuators per channel, as illustrated in Figure 6. Each motor is driven by a half-wave signal. This type of transmission minimizes the average tension since only one tendon is stressed when force is transmitted. Seen as noise on the force signal, friction will grow with the intensity of the transmitted force. The mechanical signal-to-noise ratio is roughly constant across its dynamics range instead of decreasing with the signal intensity as in a conventional transmission. 
ASME J. of Dynamics Systems, Meas. and Contr. 120(2):241--249. 1998.

A current amplifier inverts by feedback the electrical transfer function (roughly an RL circuit) of the actuators and insures that current, and therefore torque, tracks precisely the input control signal across a bandwidth much larger than the mechanical bandwidth of the drive. Displacement and force are measured directly on the tendon path via optical sensors developed in our laboratory.

\subsection{Approximate Model}

The input-output behavior (from $F_{\tau}$ to $F_{m}$ ) of the tendon transmission exhibits a complex hysteretic-like behavior. See Figure 7 for a input-output phase plot measured at low frequency.

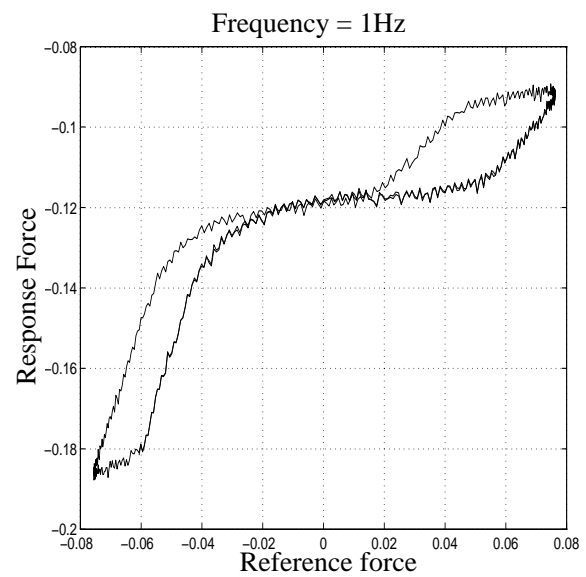

Figure 7: Hysteretic behavior of the plant.

Such a system may be viewed as the combination of sub-systems, which once combined create an apparently complicated behavior. It includes a linear sub-system representing elasticity, damping and inertia of its mechanical components which combine to form a low pass filter. The empirical transfer function of the system, obtained with a stiff load is well defined and does not change with time. It includes a resonant characteristics that can be precisely identified for a given input amplitude, but which shifts from $15 \mathrm{~Hz}$ to $35 \mathrm{~Hz}$ depending on the amplitude of the input, due to nonlinearities, as shown on Figure 8.

It was verified that nonlinear stiffening of the material used to make the tendons was not significant. The cause for a nonlinear response is friction. Friction has been extensively studied and various models have been proposed. The reader is referred to the extensive survey by Armstrong-Hélouvry et al. (1994) for a summary.

We adopt the simplest representation of friction: the "breakaway" model. With this model, the system is viewed to transmit torque (or force), while motion is not considered. The input torque is transmitted to an output torque minus a torque lost in dissipation. When the input torque is under a threshold (under the breakaway level), no torque is transmitted to the output so the friction balances exactly the input torque. This results in an input-output force-force friction model represented as a dead-band as shown on Figure 9, which is a single valued relationship. The cascade of a linear filter with a nonlinear single valued relationship results in a so-called Weiner model. 
ASME J. of Dynamics Systems, Meas. and Contr. 120(2):241--249. 1998.
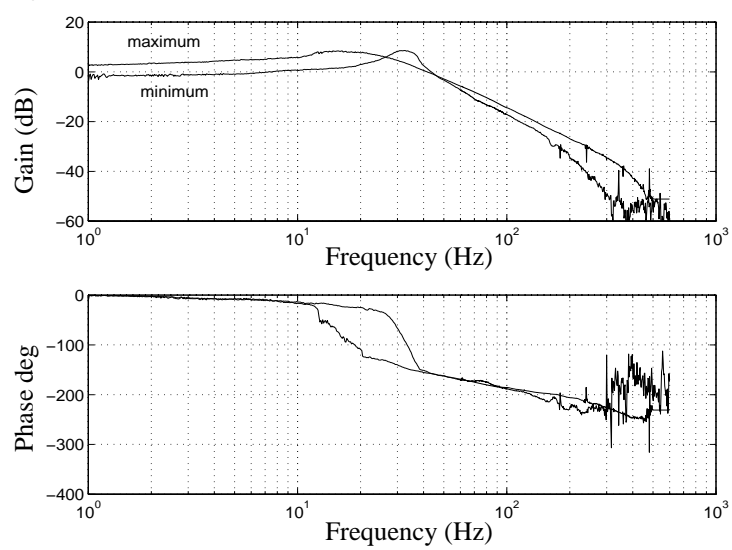

Figure 8: Open loop Bode plot.

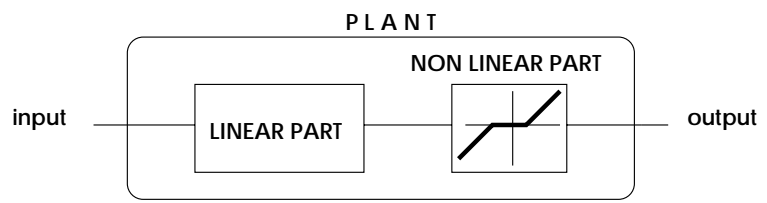

Figure 9: Plant representation: Wiener model (Wiener, 1958)

Such a cascade will create a hysteretic-like behavior. Considering a slow ramping input: while in the deadband, no signal is observed at the output; this has the effect of shifting the response on the right. When the input reverses, the system enters the deadband again, shifting the response to the left, and so on, forming a hysteresis-like loop with the input-output relationship forking at each reversal of the input.

In order to verify that the transfer function actually decomposes in the needed fashion, a linear model $\hat{G}_{L}(s)$ of the plant is identified (using conventional identification methods) and the same input is presented to the plant and to the model. The phase plot of the output of the plant is traced against the output of the model using a set-up illustrated on Figure 10. The optimal model $\hat{G}_{L}(s)$ (Equation (11)) will minimize the area of the phase plot at all frequencies and amplitudes. See Figure 11 for the experimental result.

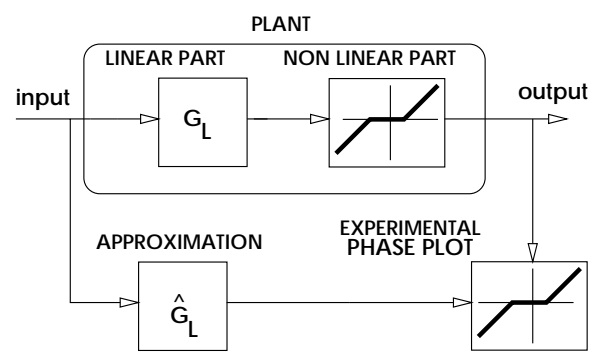

Figure 10: Block diagram representation of the input-output phase plot 
ASME J. of Dynamics Systems, Meas. and Contr. 120(2):241--249. 1998.

$$
\hat{G}(s)=\frac{35530.57}{s^{2}+56.54 s+35530.57}
$$

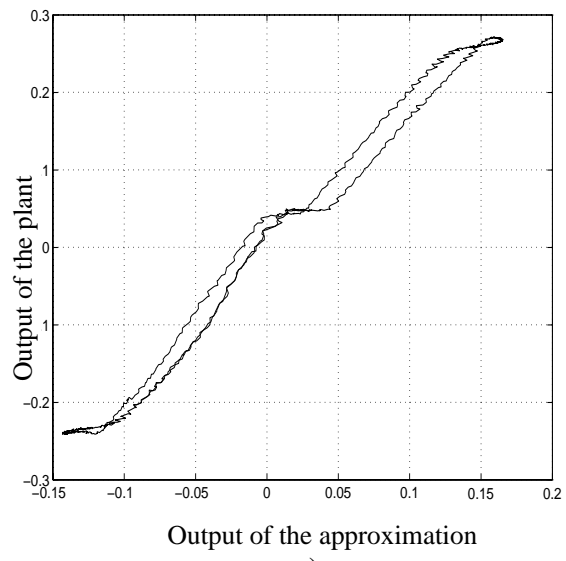

a)

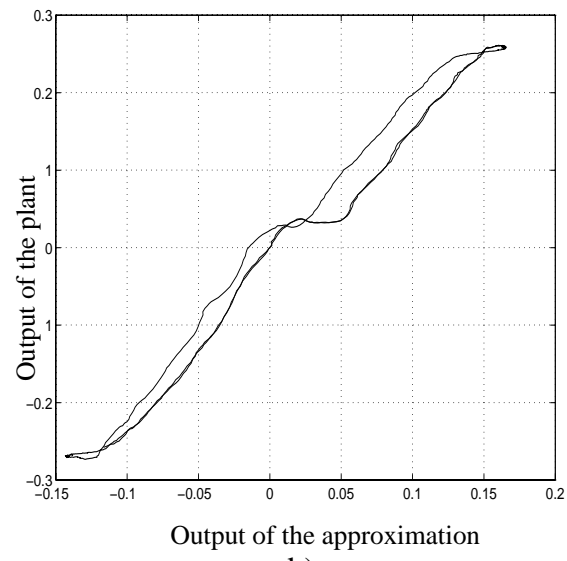

b)

Figure 11: Experimental input-output phase plots taken with input frequencies a) $0.2 \mathrm{~Hz}$. , and b) $1 \mathrm{~Hz}$.

\subsection{Controller Synthesis}

The primary objective of the controller is to extend the frequency response to the widest range possible. One way to achieve this is to stiffen the transmission by compensation with the following expected consequences.

- Stiffening should have the effect of reducing the hysteretic-like behavior of the plant.

- Arbitrary controllers having dynamics, for example a PD, a PI, or any other filter, are liable to create complex behaviors including instabilities, limit cycles, or even chaotic patterns (Towsend and Salisbury, 1987, Armstrong-Hélouvry et al., 1994). A stiffening controller should minimize these effects.

- The increase of the closed-loop stiffness of the transmission should result in a reduction of the sensitivity to the load.

A loop shaped controller is based on an approximate inversion of the plant in order to achieve a desired response. For a well chosen response, it will result in stiffening the transmission in the largest frequency range the plant can achieve.

Two controllers are designed and experimented with: a proportional controller for reference and comparison, and a loop shaped controller.

\subsubsection{Proportional Controller}

The tuning involves choosing a phase margin and selecting the highest gain possible. The experimental closed loop Bode plot is shown in Figure 12. As predicted, it has a marked resonant characteristic. An effective apparent friction reduction is achieved. The usable frequency range, which was $40 \mathrm{~Hz}$ open loop, is slightly improved. It is robust and noise free. 
ASME J. of Dynamics Systems, Meas. and Contr. 120(2):241--249. 1998.
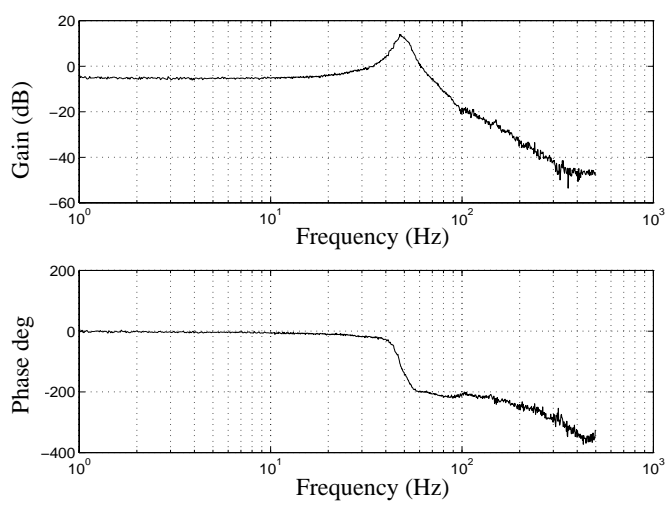

Figure 12: Closed loop Bode plot of the force response with a proportional controller.

\subsubsection{Loop Shaped Controller}

Loopshaping is applied to design a feedforward controller (Doyle et al., 1992). The idea is to choose a loop transfer function $L(s)$ so that we can achieve a robust performance, good robustness with $T(s)$ small at high frequencies, and disturbance rejection at low frequencies making $S_{G}^{T}$ small, which is not possible to achieve just by modifying the system parameters as we have shown in Section 3. The condition to design a controller with robust performance are to have an internally stable plant and to enforce the following inequality:

$$
||\left|W_{1} S\right|+\left|W_{2} T\right| \|_{\infty}<1
$$

$W_{1}$ is a weighting function used to determine internal stability by enforcing nominal performance such that $\left\|W_{1} S\right\|_{\infty}<\epsilon$, where $\epsilon$ is the maximum amplitude of the error signal over the whole frequency range. $W_{2}$ is another weighting function to enforce robust stability, $\left\|W_{2} T\right\|_{\infty}<1 . T$ is the closed loop transfer function and $S$ the sensitivity function $S_{G}^{T} . L(s)$ can then be determined using a graphical method.

The controller $C(s)$ is obtained from $C(s)=\frac{L(s)}{P(s)}$, with $P(s)=\hat{G}(s)$. The controller has to be proper and internal stability of the plant has to be ensured. This method is suitable for our purpose since the plant $G(s)$ is stable and minimum phase, as can be seen in Figure 8, and has all its poles and zeros in the right half plane.

Referring to Figure 10, the objective is to cancel the low pass dynamics of the plant in order to achieve stiffening, so that the feedback will only see a univalued input output relationship in the frequency range the plant can achieve. This concept is represented in Figure 13. Given a model $\hat{G}$, the ideal controller is simply $\hat{G}_{L}^{-1}(s)$; however, since the plant is low pass, it would not have a proper transfer function and would not be realizable. We must therefore settle for an approximate inverse in the desired frequency range and poles are added to achieve this.

We chose $L(s)$ to behave as a second order system of the form:

$$
L(s)=\frac{\omega_{n}^{2}}{s^{2}+2 \zeta \omega_{n} s+\omega_{n}^{2}}
$$

with a natural frequency $\omega_{n}=40 \mathrm{~Hz}$, and $\zeta=0.5$. This is a good response in open loop and is what we can expect for this plant. Physically, the plant cannot achieve more than a few Hertz 
ASME J. of Dynamics Systems, Meas. and Contr. 120(2):241--249. 1998.

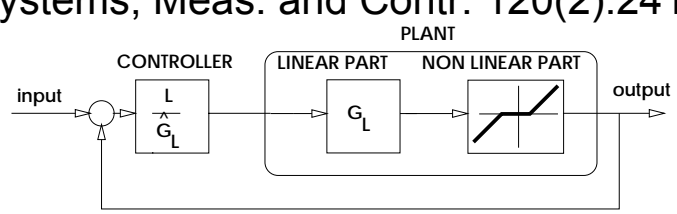

Figure 13: Loopshaping technique.

beyond its original natural frequency, for this reason we did not place the response of $L(s)$ further than 40Hz. Moreover, in closed loop the bandwidth will increase. As we demonstrated experimentally, we can achieve almost the same bandwidth for an $L(s)$ with $\omega_{n}=40 \mathrm{~Hz}$ than for $\omega_{n}=80 \mathrm{~Hz}$. The difference is that the closed loop response has dithering behavior when we used a higher $\omega_{n}$. This happens because the noise that appears after $40 \mathrm{~Hz}$ is amplified, and because we were breaking the condition needed to achieve a robust performance as specified by the loopshaping technique.
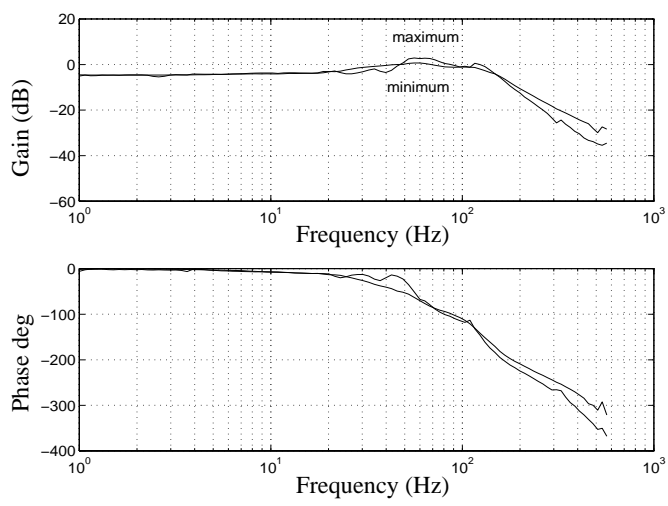

Figure 14: Closed loop Bode plot using $C(s)=\frac{L(s)}{P(s)}$.

The controller was designed using an approximation to the plant obtained with a large input amplitude, when nonlinear disturbance is minimized. It is nevertheless effective for all amplitudes. The experimental response of the system in closed loop using the controller described above is presented in Figure 14. No matter which amplitude is applied to the input of the closed loop system, the result is always almost the same. The range of uncertainty in amplitude (Figure 8), which was due to friction was quite large, but this range has been reduced to a negligible level. The bandwidth of the system which was never better than $20 \mathrm{~Hz}$ (for a phase shift of $-180^{\circ}$ ) is now extended to $150 \mathrm{~Hz}$.

Finally, we can inspect the input-output phase plot of the closed loop system and compare it to the original open loop behavior, for two different frequencies and amplitudes as in Figure 15. The deadband presented in open loop as well as the hysteresis-like behavior is corrected to give a linear behavior. The small area that appears in the closed loop signal is due to some phase shift between the input and the output and is not due to hysteresis. We have been able to improve the system response and compensate for nonlinearities, not just for some frequencies and amplitudes but for a wide range of them. 
ASME J. of Dynamics Systems, Meas. and Contr. 120(2):241--249. 1998.

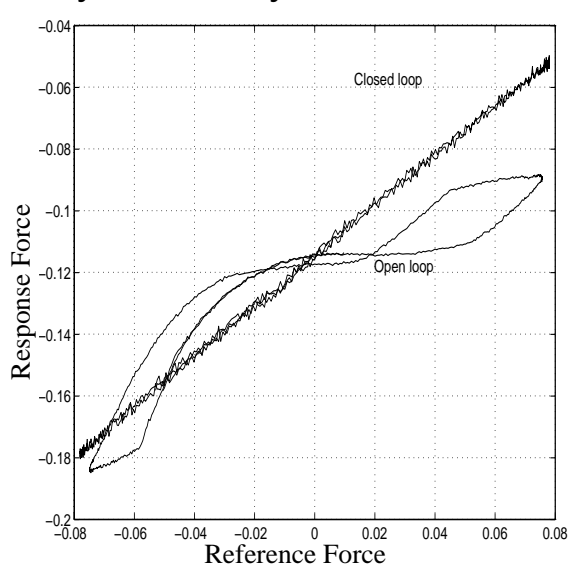

a)

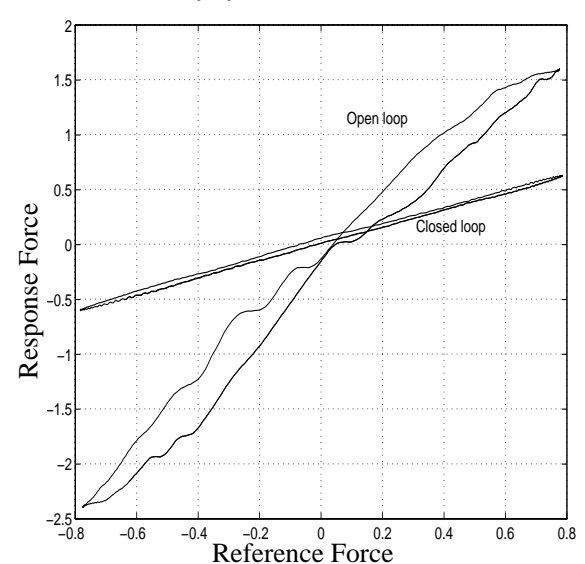

b)

Figure 15: Comparison of open and closed loop. a) Small amplitude with F=0.2 Hz., and b) Large amplitude with $\mathrm{F}=1 \mathrm{~Hz}$.

\section{Conclusion}

A model for force transmission systems was presented. A sensitivity analysis was carried out to understand how the parameters affect the behavior of the system. It was found that for the value of $r$, a tradeoff between friction rejection, inertia reduction and saturation in the actuator has to be achieved. $k_{e}$ (the force dividing factor) was found to be an expression of the degree of collocation of the force sensor along the transmission. Sensitivity analysis enable us to show the effects of collocation on a transmission ability to transmit forces. The effect of a variable load $k_{z}$ can be reduced only by a feedback controller. The proportional controller is the only one which can reduce the sensitivity function $S_{G}^{T}$, without any complicated tuning.

The criteria to choose an appropriate approximation of the plant was presented. The final design of the controller for this plant was done using the loopshaping technique. This controller, because of its robustness and disturbance rejection, compensates for the nonlinearities that appeared in open loop and also reduces the uncertainty range of the response.

\section{Acknowledgements}

Initial funding for this project was provided by a research contract with the Canadian Space Agency (No. 9F009-1-1441/01-SR). Funding is now provided by the project "Haptic Interfaces for Teleoperation and Virtual Environments" (HMI-6) funded by IRIS (second phase), the Institute for Robotics and Intelligent Systems part of Canada's National Centers of Excellence program (NCE). Additional funding was from an operating grant from NSERC, the National Science and Engineering Council of Canada.

The second author would like to acknowledge the generous support of Universidad Nacional Autónoma de México (UNAM) in the form of a postgraduate fellowship.

The authors wish to acknowledge contributions of Christopher Strong, Xianze Chen, and Ian Sinclair from MPB technologies Inc., Montreal, Canada. 
Armstrong-Helouvry, B., Dupont, P., and Canudas De Wit, C., 1994, "Survey of models, analysis tools and compensation methods for the control of machines with friction", Automatica, Vol. 30(7), pp. 1083-1138.

Doyle, J. C., Francis, B. A., and Tannenbaum, A. R., 1992, Feedback Control Theory, New York: Maxwell Macmillan International.

Eppinger, S. D., and Seering, W. P., 1987, "Understanding Bandwidth Limitations in Robot Force Control", Proc. IEEE International Conference on Robotics and Automation, Vol. 1, pp. 904-909.

Frank, P. M., 1978, Introduction to sensitivity analysis, Academic Press.

Guo, L.K. and Zhang Q.X. 1988. "The Sensitivity Analysis of dynamic Parameters in Robot Dynamics", Proc. International Conference on Systems, Man and Cybernetics, Vol 1, pp. 94-97.

Hayward, V., 1995, "Toward a seven axis haptic interface”, IROS'95, Int. Workshop on Intelligent Robots and Systems, pp. 133-139.

Jacobsen, S. C., Ko, H., Iversen, E. K., and Davis, C. C., 1989, "Control Strategies for Tendon-Driven Manipulators", Proc. International Conference on Robotics and Automation, Vol. 1, pp. 23-28.

Jen, C.W., and Johnson, D.A. 1991 "Modal Sensitivity Analysis of Planar Robots, Based on a Simplified CMS Approach", Journal of Robotic Systems, Vol. 8, No. 4, pp. 443-463.

Kazerooni, H., 1988. "Compliance control and stability analysis of cooperating robot manipulators", Robotica, Vol. 7, pp. 191-198.

Townsend, W. T., and Salisbury, S. K., 1987, "The effect of Coulomb friction and stiction on force control", Proc. IEEE Conference on Robotics and Automation, pp. 883-889.

Wiener, N., 1958, Nonlinear problems in random theory, New York: The Technology Press of The Massachusetts Institute of Technology and John Wiley and Sons, Inc.

Wierzbicki, A., 1984, Models and sensitivity of Control Systems. Elsevier science publishing company, Inc. 